\title{
Informational masking and auditory attention
}

\author{
MARJORIE R. LEEK \\ Walter Reed Army Medical Center, Washington, D.C. \\ and \\ MARY E. BROWN and MICHAEL F. DORMAN \\ Arizona State University, Tempe, Arizona
}

\begin{abstract}
Informational masking is broadly defined as a degradation of auditory detection or discrimination of a signal embedded in a context of other similar sounds; it is not related to energetic masking caused by physical interactions between signal and masker. In this paper, we report a systematic release from informational masking of a target tone in a nine-tone rapid auditory sequence as the target is increasingly isolated in frequency or intensity from the remaining sequence com. ponents. Improved target-tone frequency difference limens as isolation increases are interpreted as a reflection of increasingly focused auditory attention. The change from diffuse to highly focused attention is gradual over the frequency and intensity ranges examined, with each 1-dB increment in target intensity relative to the remaining components producing performance im. provements equivalent to those produced by a $2 \%$ increase in frequency isolation. The results are modeled as bands of attention in the frequency and intensity domains. For attention directed by frequency isolation, there is a strong correspondence with auditory filters predicted by the power spectrum model of masking. These data also support the existence of an attention band of intensity, with a bandwidth of about $5-7 \mathrm{~dB}$ at the moderate levels used in this experiment.
\end{abstract}

The ability to hear small acoustic changes in tones embedded within rapid auditory patterns can be improved significantly when the focus of auditory attention is optimally placed. Watson and his colleagues have shown that this can be accomplished through consistent practice with a pattern that is constant from trial to trial-a condition of minimal stimulus uncertainty (Watson, Kelly, \& Wroton, 1976). Through training, listeners learn to ignore irrelevant portions of an auditory pattern and focus on the target portion only.

In this study, the focus of auditory attention was manipulated not through training, but through alteration of the frequency or intensity relationships between a target tone and its neighboring tones. The degree of attentional focus was inferred from target-tone frequency difference limens (DLs) measured in several conditions of frequency and intensity isolation of the target from the remaining pattern components. Increasing isolation along either dimension allowed finer resolution of changes in the frequency of the target tone.

An improvement in target resolution may be considered a release from informational masking. Watson et al.

Support for this research was provided in part by a grant from the National Institute on Deafness and Other Communication Disorders. The opinions or assertions contained herein are the private views of the authors and are not to be construed as official or as reflecting the views of the Department of the Army or the Department of Defense. We wish to thank Bertram Scharf and two anonymous reviewers for valuable comments on an earlier version of this paper. Requests for reprints should be sent to Marjorie R. Leek, Army Audiology and Speech Center, Walter Reed Army Medical Center, Washington, DC 20307-5001.
(1976), following Pollack (1975), used this term to describe the degradation of discrimination or detection thresholds of one sound in the presence of another that is not attributable to the energy relationship of the two sounds-that is, energetic masking. Informational masking is tied to a listener's ability to " find" the portion of a complex sound that contains the information necessary for successful completion of an experimental task. Leek and Watson (1984) reported that one successful strategy used by listeners to improve resolution was to focus on a particular spectrotemporal region in a pattern to the exclusion of the remaining portions. This is a type of "spotlight" strategy; that is, if the observer's focus is directed toward the correct tone, detection or discrimination may proceed at levels approaching those seen when the target is presented without any interfering context. Duncan (1984) hypothesized a similar process in the visual attentional system. He suggested that, in the analysis of a complex array of visual information, an initial preattentive processing stage directs the observer's focus of attention, subsequently permitting a detailed description of a target to be reported.

Improvements in target resolution have been demonstrated when auditory attention is focused by a number of techniques (for review, see Leek, 1986). These include minimal uncertainty training as described by Watson et al. (1976), as well as methods of providing cues to the frequency or temporal location of a target. Cues presented to the contralateral ear (see, e.g., Taylor \& Forbes, 1969) and those provided by the contextual tones in a sequence (Howard, O'Toole, Parasuraman, \& Bennett, 1984) have both shown some success in reducing masking. 
Possibly the strongest and most compelling cue to the optimal placement of attentional focus is provided by the degree of acoustic similarity between the target and remaining components. A target that is sufficiently different from the surrounding tones along some acoustic dimension will be heard with increased precision. In a high stimulus uncertainty task (patterns changing on every trial), Spiegel and Watson (1981) observed that an increase of target intensity of about $15 \mathrm{~dB}$ relative to the remaining tones in a sequence provided as much advantage in target tone frequency discrimination as that provided by extensive training with an unchanging pattern. That is, the effects of minimal-uncertainty training could be matched by increasing the level of the target tone in a high uncertainty condition. Leek and Watson (1984) used an increase in target duration as a training technique to assist listeners in detecting the presence of a target tone embedded in a pattern. The success of this technique may have been due to the duration differences between target and context tones.

McBride (1986) reported improvements in frequency discrimination of an embedded target tone when it was in a frequency region distinct from the remaining tones. Perceptual isolation in the frequency domain proved to be a powerful influence on target discrimination, improving performance when the isolated tone was the target, but interfering when the technique provided a false cue to the target.

The attentional effects reported in that study are related to auditory stream segregation, studied extensively by Bregman and his colleagues (e.g., Bregman \& Campbell, 1971; Bregman \& Rudnicky, 1975; Tougas \& Bregman, 1990). Bregman and Campbell (1971) reported that within a rapid tonal sequence, the temporal order of components similar in frequency was correctly perceived by listeners, whereas temporal order information was lost when tones were widely separated in frequency. Bregman (1981) attributed this phenomenon to the tendency for like-frequency components to form into groups. Auditory analysis is relatively precise within groups, but difficult across groups. A similar auditory phenomenon, termed temporal coherence, was studied by van Noorden (1975) with segregation by intensity as well as by frequency. Both of these investigators found that there were combinations of temporal, frequency, and intensity characteristics of patterns that divided the auditory range into three regions-one where all components of a pattern stayed together in one group (temporal coherence, or one stream), one where a subgroup of components was perceived as a separate auditory 'object"' (segregation or temporal fission), and an ambiguous region where both percepts were reported, sometimes depending on the attentional "set" of the listener. Perception in this ambiguous region has been likened to the figure/ground phenomenon for visual stimuli, in which sometimes a portion of a picture can be seen as the figure, whereas with the blink of the eyes and a cognitive shift, it would be seen as the background.
Although there are reports of improved target resolution when any acoustic dimension provides a cue isolating the target (Leek, 1986), there has been little attempt to quantify the levels of difference required for equal performance that would indicate equivalent levels of attentional focus. In this study, the availability of cues in the stimulus patterns is controlled systematically, and the value of those cues is measured in stimulus terms. The magnitude of target-tone differences in intensity or frequency resulting in focused attention was measured under conditions of moderately high stimulus uncertainty. The degree of attentional focus was inferred from frequency DLs for target tones systematically separated from the remaining sequence tones along these two dimensions. Four basic questions were addressed:

1 . What differences in frequency and in intensity between a target tone and its surrounding pattern components are sufficient for subjects to overcome informational masking?

2. What is the shape of the function relating the separation of the target in frequency or intensity and decreasing DLs? That is, is it abrupt or gradual?

3. Where do the equal-attention contours fall in the frequency/intensity space?

4. Are the effects similar in different frequency regions? A comparison of results in two different frequency regions may help to determine the contributions of peripheral auditory processing and more central cognitive processing on the direction of auditory attention.

\section{METHOD}

\section{Subjects}

Four subjects ranging in age from 21 to 34 years served as listeners. Each listener demonstrated audiometric thresholds of $15 \mathrm{~dB}$ HL (re: ANSI, 1969) or less at octave frequencies from 250 to $8000 \mathrm{~Hz}$. None of the subjects had participated in previous investigations involving tonal patterns.

\section{Stimuli}

Frequency DLs were measured for target tones embedded in rapid tonal sequences. Isolation between target and surrounding tones was manipulated along two acoustic dimensions-frequency and intensity-in two pitch regions, producing four experimental conditions. Within each condition, six patterns were constructed, each consisting of nine 46-msec tones differing from each other in frequency. Each tone included $3-\mathrm{msec}$ rise and fall times to reduce audible transients.

The construction of the six patterns for each condition reflected a systematic isolation by either frequency or intensity difference of a selected target tone from the remaining pattern components. The tonal components were arranged in a sequence with no silent intervals between them. The order of the tones was different for each of the 24 patterns and was pseudorandom, taking into account some effects known to influence pattern discrimination. The target tones in each pattern were chosen to be similar to each other, but not identical in frequency. Because targets high in frequency relative to the remaining tones and/or near the ends of sequences are easily resolved (Watson, Wroton, Kelly, \& Benbasset, 1975), these tones were always the lowest frequency in the sequence and occurred at the temporal center. In light of the finding of Watkins and Dyson 


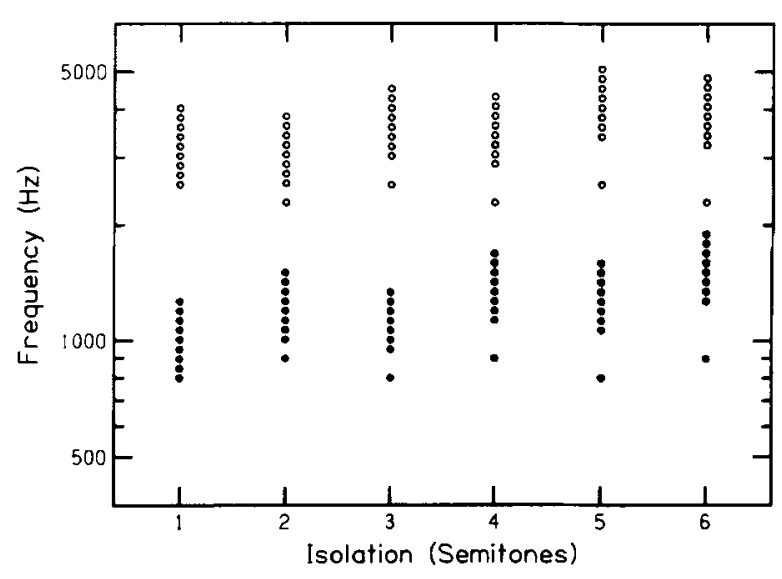

Figure 1. Frequencies in six patterns at the low pitch region (solid circles) and the high pitch region (open circles). The label on the abscissa describes the amount of frequency difference of the target tone from the context tone nearest in frequency.

(1985) that reversals in direction of frequency change within a sequence might provide a cue to a target location, the target in these patterns was always one of five such reversals in each pattern.

Isolation by frequency difference. The frequencies of the pattern components for each of the two frequency-isolation conditions are displayed in Figure 1. The lower pitch region, with frequencies ranging from 800 to $1896 \mathrm{~Hz}$, is shown with solid circles. The target tone was either 800 or $900 \mathrm{~Hz}$. The higher pitch region included frequencies from 2295 to $5074 \mathrm{~Hz}$, shown with open circles, with a target tone of either 2295 or $2550 \mathrm{~Hz}$. There was 1 semitone ( $1 / 12$ octave) between each tone in the context, and from 1 to 6 semitones separated the lowest frequency component (which was always the target) from the nearest frequency context tone. Note that as the isolation increases from 1 to 6 semitones, the target tone is increasingly separated from the remaining eight pattern tones. All tones were presented at $75 \mathrm{~dB}$ SPL.

Isolation by intensity difference. In order to isolate the target by intensity, six patterns were constructed for each pitch region with all tones, including the target tone, spaced 1 semitone apart in frequency (similar to the display in Figure 1 at 1 semitone). However, in each pattern, the intensity of the target tone varied from 75 to $85 \mathrm{~dB}$ SPL, while the remaining tones were at $75 \mathrm{~dB}$. Target tone frequencies were either 850 or $950 \mathrm{~Hz}$ in the low pitch region, with the remaining tone frequencies extending to $1503 \mathrm{~Hz}$. In the upper pitch region, the target tone was either 2433 or $2703 \mathrm{~Hz}$. with context tones up to $4276 \mathrm{~Hz}$.

\section{Apparatus}

All stimulus generation and response collection was controlled by a PDP-11/23 computer. The components of the tonal patterns were produced by a voltage-controlled oscillator (Wavetek, Model $182 \mathrm{~A}$ ), gated through a computer-controlled electronic switch, attenuated, and delivered over a Telex 1470A headphone mounted in MX-41/AR cushions. The listener, seated in a sound-treated booth, received the signal monaurally in the right ear and responded to each trial by pressing buttons on a hand-held response box. Lights on the response box visually indicated the timing of trial events and provided correct-answer feedback on each trial. Acoustic calibration of the equipment was conducted prior to each test session using a Quest (Model 215) artificial ear assembly.

\section{Procedure}

Target frequency DLs were measured for each of the 24 patterns ( 2 dimensions, 2 pitch regions, 6 isolation steps). On each trial, the listener was asked to discriminate among three presentations of a given pattern that were identical except for a small increment in target-tone frequency on one of the presentations. Pattern presentations were separated by a 300 -msec interstimulus interval. The first presentation was always the standard by which the other two presentations were judged. The listener was asked to indicate which of the two remaining presentations was different from the standard by pressing the appropriate button on the response box. There was an equal probability on each trial that the difference would occur on the second or third presentation. A 1.5-sec response time was provided for each trial. Once a selection had been made, the correct response was indicated by a feedback light on the response mechanism.

Differences in target tone frequency varied according to a threshold tracking procedure designed to seek the level that produced $71 \%$ correct discriminations (Levitt, 1971). Two successive correct responses decreased the frequency difference in target tone by a factor of 0.8 . The frequency difference increased by a factor of 1.25 for every incorrect response. Within each condition, six adaptive tracks were maintained, one for each pattern in the condition. The selection of pattern (and, therefore, of degree of isolation) was random on each trial.

Each subject participated in all four experimental conditions in the same order. First the low pitch-frequency dimension was presented as a practice run. Data from the practice run were not included in the final tabulations. Following the practice run, the order of presentation was: low pitch/intensity; high pitch/frequency; high pitch/intensity; low pitch/frequency.

Within each condition, the target-tone frequency difference was initially $80 \mathrm{~Hz}$ on each interleaved adaptive track. The first 120 trials were considered practice, to make sure that the listener understood the task and could discriminate large differences. The final frequency differences obtained from this practice block served as the initial levels for the remaining test blocks in each experimental condition. Each block of trials consisted of 120 trials, with 20 presentations of each pattern per block. Each of the six adaptive tracks was continuous over four test blocks, with brief rest periods between the blocks to reduce listener fatigue. Threshold values obtained for each of the six isolation steps (or patterns), therefore, were based on 80 trials ( 20 presentations per block $\times$ four blocks). The average number of track reversals for each track was 12 and ranged from 6 to 20 . The listeners completed an average of two conditions per experimental session, lasting about 2 hours

\section{RESULTS}

\section{Effects of Increasing Target-Tone Acoustic Isolation}

Frequency DLs were defined as the difference (in hertz) in target-tone frequency necessary to be recognized $71 \%$ of the time. For each subject in each condition, these threshold values were converted into relative measures of frequency discrimination by dividing each DL by the corresponding target-tone frequency. The geometric means and standard deviations of the relative DLs (denoted as $\Delta f / f)$ across the 4 subjects were then calculated for each isolation step along the two acoustic dimensions of frequency and intensity.

The mean threshold values for the two isolation dimensions at the two pitch regions are shown in Figure 2. The values on the abscissa represent the amount of difference in either semitones or decibels between the target tone and the remaining tones in the patterns. The top two panels show results from patterns in the low pitch region, and the bottom panels show performance on patterns in the 


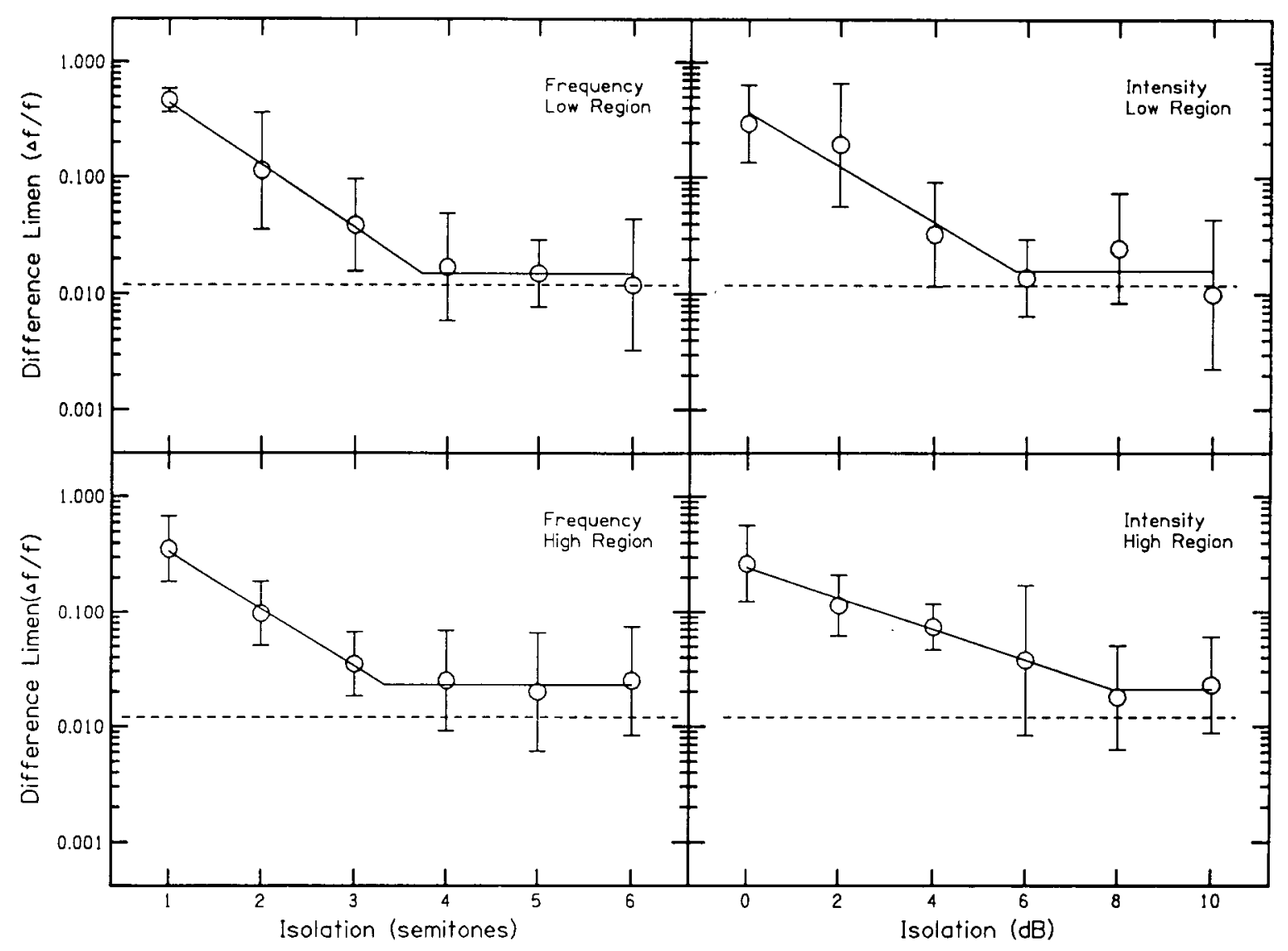

Figure 2. Relative difference limens for frequency isolation conditions on the left and intensity isolation conditions on the right. The top panels show data from the low pitch region, while the lower panels are from the high pitch region. Data points are geometric mean values for 4 subjects; error bars represent $\pm 1 S D$. The dashed line reflects performance expected if the target were a single tone. Solid lines are the best-fitting intersecting lines, with the constraint that the second line have a slope of zero.

high pitch region. The left panels show the effect of isolating the target tone along the frequency dimension, and panels on the right reflect performance with isolation along the intensity dimension. Error bars represent one standard deviation around the mean values. The dashed line on each panel of Figure 2, taken from Leek and Colleran (1984), indicates the mean frequency DL for a single 46-msec, $920-\mathrm{Hz}$ tone presented to 10 experimentally naive listeners. This value may be viewed as an estimate of the limit of performance by the present group of listeners if there were no tones surrounding the target tone.

Figure 2 indicates that, in all conditions, frequency DLs were large when the target tone was very close in frequency or intensity to the remaining tones in a pattern. DLs decreased as the target tone was isolated from the surrounding tones. Once the target tone was sufficiently different from the other tones, the subjects appeared to be able to "hear out" the target rather easily, and performance tended to approach asymptotic levels. For both acoustic dimensions and both high and low pitch regions, these asymptotic values were similar to those obtained by Leek and Colleran (1984) for single tones, suggesting that little further improvement in performance would be produced by greater degrees of acoustic isolation.

The solid lines in Figure 2 are two best-fitting lines to the mean data points, with the constraint that the second line must have a slope of zero. These lines were fit using a procedure described by Rabinowitz, Bilger, Trahiotis, and Nuetzel (1980) based on a method first presented by Bogartz (1968). Candidate two-line combinations are determined in an iterative manner by a least-squares method with a systematic assignment of the data points to each line. For each pair of lines, the variance of the data accounted for by the fits, $R^{2}$, was calculated. The lines selected as best describing the data are those that maximize $R^{2}$. The intersection of the two lines is assumed to be the minimum degree of isolation required to segregate the target tone for the most precise resolution. The intercept of the flat line is an estimate of the asymptotic performance for each condition. A similar analysis was carried out for the individual data and will be described subsequently.

The fits were more precise for the frequency conditions (mean $R^{2}=.99$ ) than for the intensity conditions (mean 
$R^{2}=.93$ ). Although asymptotic thresholds tended to be slightly higher in the upper pitch regions than in the lower pitch regions, the differences were small. Terminal threshold values for the two isolation dimensions were, however, quite similar within pitch regions.

To determine whether the results shown in Figure 2 represent significant differences across isolation dimension, pitch region, or degree of acoustic isolation, a three-way analysis of variance with repeated measures was performed on the logarithms of the relative frequency DLs. There was a significant effect of isolation magnitude $[F(5,15)=35.73, p<.0001]$, but no other main effects or interactions were significant (all $p s>.10$ ). There were, therefore, no systematic differences across these subjects in how the stimulus pattern characteristics influenced the ability to recognize small changes in the target tone, the exception being the amount of acoustic isolation of the target.

\section{Relationship Between Acoustic Isolation by Frequency and Intensity}

As in most studies of complex sound processing, there were large differences in overall performance of the subjects, although for all listeners, DLs tended to decrease with increasing target separation. Furthermore, performance within subjects for the two acoustic isolation dimensions was highly correlated, as is shown in the scatterplots in Figure 3. Each point on the plots represents the relative DLs due to isolation by frequency and intensity for each subject on each of the six different isolation steps. For example, performance with 1-semitone frequency isolation is plotted against performance with a
0 -dB intensity isolation. The correlations for the low and high pitch regions were .89 and .84 , respectively.

\section{Attentional Equivalences Across Acoustic Dimension and Pitch Region}

One of our major goals in this project was to determine the equivalent benefit across the two acoustic dimensions in terms of improvements in perception. The data for all listeners and conditions suggest that performance gradually improves as the target tone becomes acoustically isolated from the remaining pattern tones, and then reaches a point at which increasing target tone isolation produces no further improvement. To quantify this description, the individual data were fit with functions providing estimates of asymptotic value, rate of improvement with increasing acoustic isolation, and degree of isolation necessary to achieve acoustic segregation (i.e., when the asymptotic value was approached). The best-fitting pair of lines was determined as described earlier for the mean data, with the slope of the second line constrained to be zero. This is a concession to the model described above, which postulates that performance approaches asymptote at the largest isolation magnitudes. This constraint produced only a small reduction in the quality of the fit over a two-line fit allowing the slopes of both lines to vary (mean $R^{2}=$ .84 vs. .87 ), suggesting that most functions were adequately fit by a zero-slope second line. Not unexpectedly, fits to individual data were generally poorer than fits to the mean data, ranging from $R^{2} s$ of .79 to .99 for the frequency isolation data, and from .52 to .92 for intensity isolation. One of the functions had a severe nonmonotonicity, resulting in an unrealistic description of the data
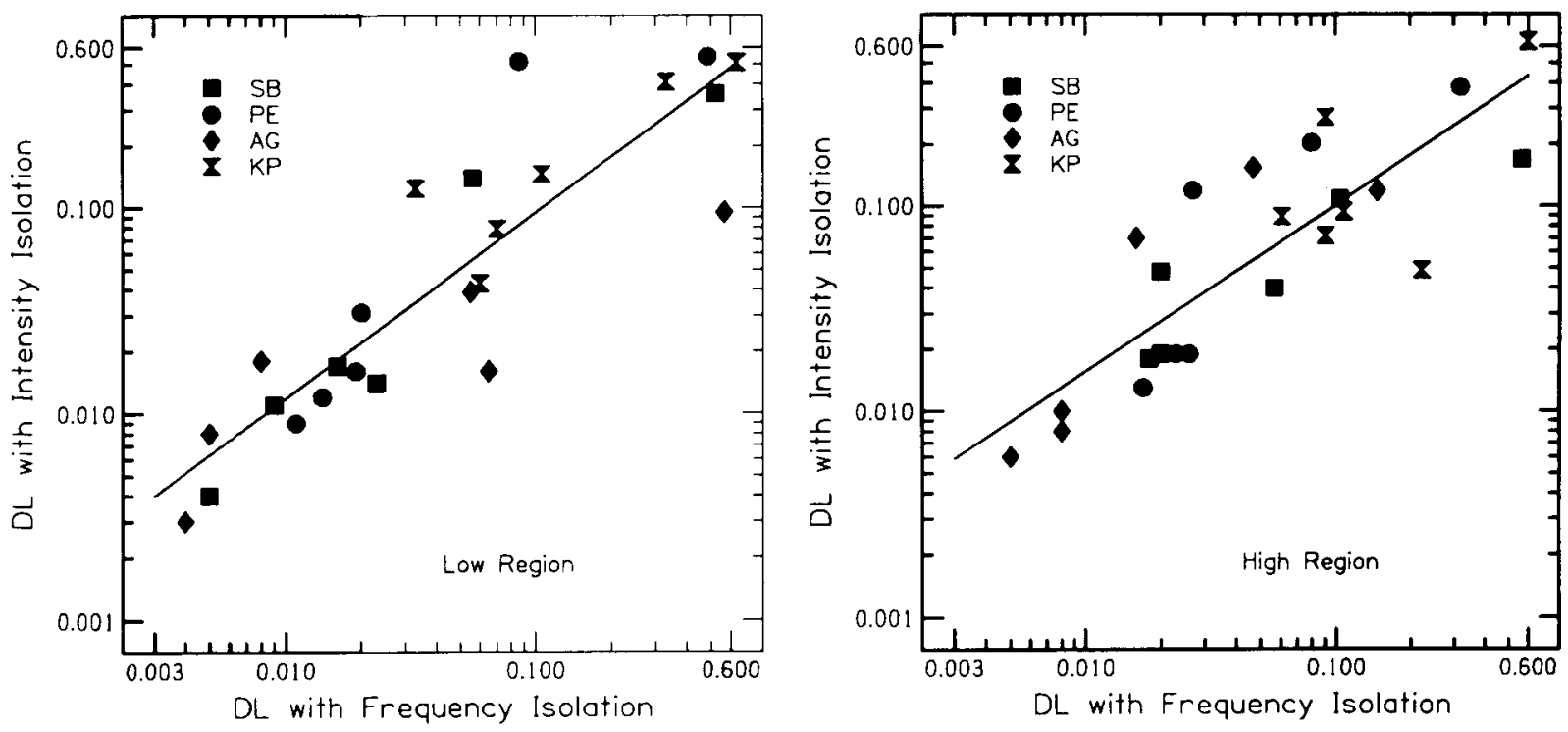

Figure 3. Individual subject data showing difference limens due to frequency isolation plotted against performance due to intensity isolation. 
Table 1

Points of Intersection of Two Best-Fitting Lines Describing Individual Subject Performance Functions

\begin{tabular}{|c|c|c|c|c|c|c|}
\hline \multirow[b]{3}{*}{ Subject } & \multicolumn{4}{|c|}{ Frequency } & \multicolumn{2}{|c|}{ Intensity } \\
\hline & \multicolumn{2}{|c|}{ Low Pitch Region } & \multicolumn{2}{|c|}{ High Pitch Region } & \multirow{2}{*}{$\begin{array}{c}\text { Low Pitch Region } \\
\text { Isolation } \\
\text { (dB) }\end{array}$} & \multirow{2}{*}{$\begin{array}{c}\text { High Pitch Region } \\
\text { Isolation } \\
\text { (dB) }\end{array}$} \\
\hline & $\begin{array}{c}\text { Isolation } \\
\text { (Semitones) }\end{array}$ & $\begin{array}{l}\text { \% Frequency } \\
\text { Difference }\end{array}$ & $\begin{array}{l}\text { Isolation } \\
\text { (Semitones) }\end{array}$ & $\begin{array}{c}\text { \% Frequency } \\
\text { Difference }\end{array}$ & & \\
\hline S.B. & 3.17 & 20 & 3.79 & 24 & 7.52 & 8.98 \\
\hline P.E. & 3.18 & 20 & 3.14 & 20 & 5.97 & 8.44 \\
\hline A.G. & 4.14 & 27 & 3.76 & 24 & 6.06 & 7.48 \\
\hline K.P. & 4.50 & 30 & 2.96 & 18 & 4.63 & 9.13 \\
\hline$M$ & 3.75 & 24 & 3.41 & 22 & 6.05 & 8.51 \\
\hline
\end{tabular}

Note-The slope of the second line is constrained to be zero.

by the best-fitting lines. In that case, only five of the six data points were fit, producing a small increase in the variance accounted for and a more sensible fitted function.

Characteristics of the fits to the individual data are shown in Tables 1 through 3 . The point of intersection of the two lines provides an estimate of the amount of acoustic isolation necessary to produce complete attentional focus on the target tone. These values are shown in Table 1 for each subject and condition. For the frequency isolation conditions, values ranged from 3.2 to 4.5 semitones for the low pitch region, and from 3.0 to 3.8 semitones for the high pitch region. These values may be translated to show that this change in performance occurred on the average for differences in frequency between target tones and surrounding context of about $22 \%-24 \%$. Pitch region had no systematic effect across subjects on the crosspoint for frequency isolation. In the intensity conditions, values ranged from 4.6 to $7.5 \mathrm{~dB}$ for the low pitch region and from about 7.5 to $9.1 \mathrm{~dB}$ for the higher pitch region. The higher pitch region always required greater intensity isolation than did the lower pitch region.

Table 2

Slopes of First Line, Indicating Change in Performance with Change in Amount of Isolation

\begin{tabular}{lccccc}
\hline & \multicolumn{2}{c}{ Frequency } & & \multicolumn{2}{c}{ Intensity } \\
\cline { 2 - 3 } \cline { 5 - 6 } Subject & Low Region & High Region & & Low Region & High Region \\
\hline S.B. & -.76 & -.50 & & -.21 & -.10 \\
P.E. & -.69 & -.54 & & -.31 & -.14 \\
A.G. & -.61 & -.48 & & -.18 & -.19 \\
K.P. & -.30 & -.43 & & -.17 & -.08 \\
$M$ & -.59 & -.49 & & -.22 & -.13 \\
\hline
\end{tabular}

Note-Units are $(\log \Delta f / f) /$ semitone for frequency and $(\log \Delta f / f) / \mathrm{dB}$ for intensity.

Table 3

$y$ Points of Intersection of the Best-Fitting Lines for Individual Subject Performance Functions

\begin{tabular}{lccccc}
\hline & \multicolumn{2}{c}{ Frequency } & & \multicolumn{2}{c}{ Intensity } \\
\cline { 2 - 3 } \cline { 5 - 6 } Subject & Low Region & High Region & & Low Region & High Region \\
\hline S.B. & .010 & .019 & & .010 & .018 \\
P.E. & .014 & .022 & & .012 & .027 \\
A.G. & .006 & .007 & & .007 & .008 \\
K.P. & .048 & .086 & & .099 & .082 \\
$M$ & .014 & .022 & & .017 & .024 \\
\hline
\end{tabular}

Note-Units are relative frequency difference limens $(\Delta f / f) . M=$ geometric mean.
The gradual increase in the focus of attention may be inferred from the slope of the first fitted line of each pair, as discrimination performance improved. These values are shown in Table 2. The slopes are shallower for the intensity dimension than for the frequency dimension, reflecting the inexact equivalence between the units of measurement for the two dimensions. The mean slope values shown in this table may be used to estimate the equivalent units of the two dimensions influencing the focus of auditory attention. The ratio of the slope values for the two dimensions at each pitch region, along with conversion of the semitones to percentages, reveals that at the low pitch region, a $2 \%$ increment in frequency isolation corresponds to a $1-\mathrm{dB}$ intensity isolation. The value at the higher pitch region is only slightly different: a $1.5 \%$ frequency difference corresponds to $1 \mathrm{~dB}$. In general, then, at either pitch region, the degree of attentional focus attributable to a $2 \%$ difference in frequency between a target tone and the remainder of the complex sound may be matched by a $1-\mathrm{dB}$ level increment in the target.

Table 3 indicates the asymptotic levels of frequency discrimination performance estimated by the intercept of the horizontal line. These scores represent an estimate of the best performance of each subject on each condition, and they should be similar across conditions if the experimental manipulations (isolation dimension and pitch region) have similar effects on performance. In fact, a two-factor repeated measures analysis of variance (dimension and pitch region) shows that these values were significantly smaller for the low pitch region than for the higher one $(p<.05)$. Within pitch region, asymptotic values for the two isolation dimensions were similar, and the interaction between pitch and dimension was not significant.

This measure also shows the two extremes of individual performance often seen in studies of complex sound perception. Subject A.G. usually produced very small asymptotic frequency DLs, whereas Subject K.P. consistently required much larger differences.

\section{DISCUSSION}

\section{Attention and Perceptual Isolation}

A major purpose of this research was to determine the magnitudes of intensity and frequency isolation of a tone embedded in an auditory pattern that resulted in equal performance on a frequency discrimination task, and by in- 
ference, equal attentional focus. Although the slopes of the performance functions shown in Figure 2 for the less isolated targets (generally, 1-3 semitones or 0-6 dB) suggest a gradual change in focus, there is a point in each function at which performance approaches an asymptote and little further improvement is produced. This point probably reflects the change from diffuse to focused attention. Perceptual isolation, as indicated by the approach to asymptotic DLs, occurred when the target was separated from the remaining components in the pattern by 3-4 semitones, or by about 6-8 dB. These values resemble those reported by van Noorden (1975) to produce temporal fission in patterns constructed of rapidly alternating tones. Van Noorden found that the tonal sequence split into separate perceptual streams at frequency differences of approximately 2-3 semitones. For the differences between the tones in intensity rather than frequency, the streams split apart for level differences of about 2-4 dB. For both dimensions, van Noorden's results are somewhat smaller than those reported here, but it should be noted that his stimuli were simple (only two alternating tones), the task was simple (reporting whether the sequence was coherent or not), and his listeners had extensive practice listening to the sequences.

Although it is possible to describe the point in the functions where the release from informational interference became complete, the decrease in DLs with increasing isolation was not abrupt. Rather, the performance functions suggest a gradual transition in attentional focus as the target became more isolated from the remaining tones in the sequence. This finding may be related to one described in the literature involving stream segregation. Both van Noorden (1975) and Bregman and Campbell (1971) observed that when frequency differences were very large or very small, there were definite perceptions of one frequency stream or more. However, there was a middle region, where the perception was more ambiguous, and where both percepts might be heard, depending on the attentional set of the listener. The gradual decrease in frequency DLs observed in the present study may correspond to such an ambiguous area of attentional focus.

The asymptotic DLs were similar for both frequency and intensity isolation dimensions, and, at least for the lower pitch region, they were also similar to discrimination performance for tones in isolation. Watson et al. (1976) reported DLs of about $\mathbf{0 1 4}$ for well-practiced listeners in a condition of minimal uncertainty, and DLs ranging up to .140 for various conditions of intermediate uncertainty. The asymptotic values estimated here fall toward the lower end of that range, suggesting that acoustic isolation provides similar benefit to minimal-uncertainty training.

The higher pitch region resulted in slightly larger terminal DLs than those found for the lower pitch region. This measure suggests that asymptotic performance may be poorer at higher frequency regions, or that more practice is needed to produce the small DLs shown for the lower frequency region. Little research has been reported on pattern discrimination at the higher frequencies. However, relative DLs for tones in isolation are not different within the frequency ranges used here (Wier, Jesteadt, \& Green, 1977), suggesting that this difference is specific to the need to hear the target within a pattern.

There are often significant learning effects in studies of tonal sequence perception (Watson, 1980; Watson \& Foyle, 1985). Although these listeners had very little practice with this task, there were no systematic differences in asymptotic performance level from the first to the last experimental conditions in which they participated. Practice effects are most significant when the pattern is not changing from trial to trial, a minimal-uncertainty experimental procedure. In the present study, not only was the pattern randomly selected on each trial from a cata$\log$ of six, but a different catalog was presented in each condition. Thus, once the initial learning of the experimental task occurred in the practice condition, one would expect little further improvement in frequency discrimination owing to training.

\section{Informational Versus Energetic Masking}

The large effects of frequency and intensity isolation on target frequency discrimation may not be a reflection of auditory attention, but instead might simply result from peripheral effects of "energetic" temporal masking on the perception of tones in sequences. Even at suprathreshold levels, the interaction of individual components of complex sounds may change loudness. In fact, the results described here are those that would be predicted if partial masking were decreasing the loudness of targets in the minimal frequency isolation conditions, with a release from such masking as the contextual components are further separated in frequency. Furthermore, as the intensity of the target tone increases relative to the context, the loudness of the target will increase. Such changes in loudness might result in the observed changes in frequency discrimination, without recourse to attention as the mediating factor. However, a review of the literature on partial masking and on the effects of sensation level on frequency discrimination suggests that the results presented here are only minimally affected by these factors.

Scharf (1964) reported 20-30 dB partial masking of a tone by a narrow-band noise centered at the frequency of the tone. Decreasing the frequency of the tone by about 3 semitones relative to the center of the masking band decreased partial masking to 3-6 dB, with further decreases as the tone and masker were separated further. These values are for simultaneous masking and therefore would be expected to be reduced for nonsimultaneous partial masking in temporal patterns. In fact, Elmasian and his colleagues (Elmasian \& Galambos, 1975; Elmasian, Galambos, \& Bernheim, 1980) reported a small enhancement of loudness of up to $5 \mathrm{~dB}$ when one high-frequency tone preceded another by $100 \mathrm{msec}$, both at the same SPL. Thus, although partial masking may significantly reduce the loudness of a target tone when both tone and masker 
occur at the same time and frequency, there is little change in loudness with separations of the target and masker along either dimension.

In the isolation condition in this study that is most vulnerable to partial masking effects-that is, the 1-semitone isolation condition-the context tone nearest in frequency to the target occurs $92 \mathrm{msec}$ before the onset of the target tone (it is the 2 nd tone in the sequence and the target is the 5th tone). Partial masking due to this tone will be reduced because of the temporal offset. The components in this pattern that immediately precede and follow the target are, respectively, 2 and 7 semitones different from the target in frequency. Thus, due to separations in both time and frequency, the amount of partial masking acting to reduce the loudness of target tones in these patterns would be quite small.

Even if there were a decrease in target loudness or sensation level due to partial masking effects, it is unlikely that the large changes in frequency DLs demonstrated here in response to varying the amount of frequency separation of the target from the context would result. For single tones in isolation, Wier et al. (1977) report only about a 1- to 4-Hz difference in frequency DLs as sensation level decreases from 80 to $20 \mathrm{~dB}$. Even if the SL (or loudness) of the target tone in these patterns was reduced by the largest amount reported by Scharf (1964) for tone and masker coinciding in time and frequency, $30 \mathrm{~dB}$, one would expect only a small change in the DL-nothing on the order of the improvements observed here with changes in target isolation.

For the intensity isolation condition, obviously there is a change in sensation level of the target tone with increases in isolation over a 10-dB range. However, again with reference to Wier et al. (1977), this change in sensation level alone would not be expected to produce the large changes in frequency discrimination observed in the present study.

\section{An Attentional Filter}

The effects of focused attention may be modeled as an attentional filter analogous to the critical band concept that forms the basis for auditory processing. According to the power spectrum model of masking, the power in a signal required for its detection is proportional to the power in a masker passed through the same auditory filter. Patterson and his colleagues have applied this model to study the shape and characteristics of the auditory filter (see Patterson \& Moore, 1986, for review). The notched-noise measurement technique used by Patterson to measure the auditory filter is in many respects analogous to the experimental procedure here. A wideband masker is systematically moved farther and farther away from a signal to be detected along the frequency axis. As the masker moves away from the signal, less of the masker energy falls within the processing filter, and therefore the threshold of the signal drops.

The same model and calculations may be applied to the findings from the present study, with the following differences kept in mind. First, it must be remembered that the masking here is informational masking, with very little contribution from physical interference among the components of a pattern. The interfering components do not occur simultaneously with the target. Characteristics of an informational masking filter have not been developed heretofore. Second, the task here is not detection, but frequency discrimination. That is, the masking does not (necessarily) make the target more difficult to hear, but it does make it more difficult to extract the relevant information from the target.

The top panels in Figure 4 show the mean DLs from Figure 2 for the frequency dimension on the left and the intensity dimension on the right, but now fit with a rounded exponential (roex) function as described by Patterson, Nimmo-Smith, Weber, and Milroy (1982). This filter shape has been shown accurately to describe the auditory filter in the power spectrum model of auditory processing. The bottom panels show the filter itself, as calculated from the DLs according to the equation:

$$
W(g)=(1-r)(1-p g) e^{-p g}+r,
$$

where $g$ is the relative difference between target and nontarget components in either frequency (left panels) or intensity (right panels), $r$ and $p$ are parameters describing the dynamic range and bandwidth, respectively, of the filter, and the ordinate, $W(g)$, is the weighting applied as a function of separation (in frequency or intensity) between target and the remaining components. One interpretation of the weighting function is that it represents competition or interference: As the difference between target and nontarget portions increases, the interference decreases. The data collected here do not allow a determination of the possible asymmetry of such a filter, so it is shown as being symmetric.

This attentional filter might be considered a listening channel, centered on the target tone. As the pattern tones are moved away from the target, more tones are removed from within the channel, and less competing information is processed through that channel. Tones within the channel provide informational interference, while tones falling outside the channel may be ignored easily.

In the frequency dimension, the fits to the mean data provided by the roex filter are excellent, and, notably, the bandwidths of the filters correspond to equivalent rectangular bandwidths for these center frequencies calculated according to Moore and Glasberg (1983)-that is, .13 and .12 times the center frequency. This close correspondence is consistent with the notion that the auditory filter generally attributed to peripheral cochlear processing and the attentional filter described here might be one and the same. A similar concept has been proposed by Greenberg and Larkin (1968), and it has been investigated more recently by Scharf, Quigley, Aoki, Peachey, and Reeves (1987) and Hafter and Schlauch (1989). These researchers used a probe signal method to investigate the effects of expectation on the detection of low-intensity pure tones. Thresholds increased for tones removed in frequency from that which listeners expected to hear. Changes in threshold as the difference between probe frequencies and expected 

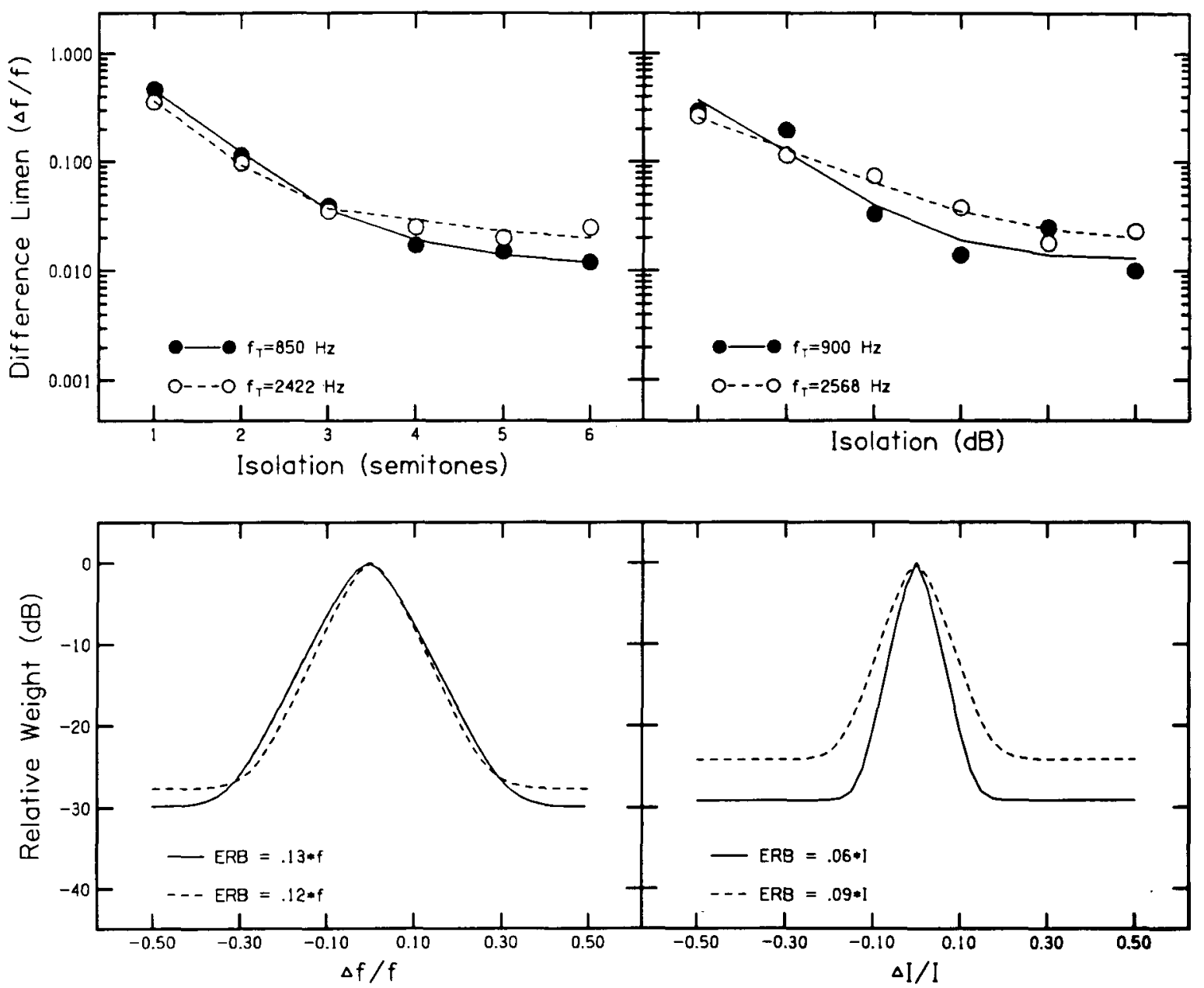

Figure 4. Top panels show mean data for frequency isolation (left) and intensity isolation (right), fit with a rounded exponential function. Bottom panels show the auditory filters calculated from the roex fits. Equivalent rectangular bandwidths (ERB) are expressed as a constant multiplied by the center frequency of the filter or by the base intensity.

frequencies increased traced a critical-band-like function termed the listening band. Hafter and Schlauch (1989) also fit a roex function to their listening-band data, and reported good correspondence with the auditory filters described by Moore and Glasberg.

The interpretation of the intensity panels on the right of Figure 4 is not quite as straightforward, nor is the fit to the data as precise. Because there are no physiological mechanisms that would underlie a filter based on intensity, van Noorden (1975) hypothesized that any such filtering was a central phenomenon. Luce and Green (1978) also supported a central locus, but argued that critical bands for frequency and what they call intensity attention bands reflect a single mechanism for selecting the part of a neural array that will be most heavily weighted by a central processor. Within an attended band, they hypothesized, neural activity is monitored maximally, whereas outside the band, neural activity is sampled much less densely. Because there is a high concentration of monitored neural activity within the attention band, acoustic information about signals located there can be extracted. Applying this logic to these data, precise frequency discrimination performance occurs when the target is within a monitored band separate from the remaining tones.

The attentional filters for intensity shown in Figure 4 have equivalent rectangular bandwidths of .06 and .09 at the low and high pitch regions, corresponding to 4.5 and $6.75 \mathrm{~dB}$ calculated from a base intensity of $75 \mathrm{~dB}$ SPL. Luce and Green (1978) suggested a bandwidth somewhat larger, 10-20 dB, and van Noorden (1975) placed the band at about $5 \mathrm{~dB}$ for these short signals. The differences among these three estimates may be related to differences in the experimental tasks. In any case, there does seem to be evidence, at least from these three studies, of a band 
of attention determined by intensity that is analogous to the critical band. This possibility would seem worthy of further investigation.

\section{REFERENCES}

BogarTZ, R. S. (1968). A least-squares method for fitting intercepting line segments to a set of data points. Psychological Bulletin, 70, 749-755.

Bregman, A. S. (1981). Asking the "what for" question in auditory perception. In M. Kubovy \& J. R. Pomerantz (Eds.), Perceptual organization (pp. 99-118). Hillsdale, NJ: Erlbaum.

Bregman, A. S., \& Campbell, J. (1971). Primary auditory stream segregation and perception of order in rapid sequences of tones. Joumal of Experimental Psychology, 89, 244-249.

Bregman, A. S., \& Rudnicky, A. I. (1975). Auditory segregation: Stream or streams? Journal of Experimental Psychology: Human Perception \& Performance, 1, 263-267.

DUNCAN, J. (1984). Selective attention and the organization of visual information. Journal of Experimental Psychology: General, 113, 501-517.

Elmasian, R., Galambos, R. (1975). Loudness enhancement: Monaural, binaural, and dichotic. Journal of the Acoustical Society of America, 58, 229-234.

Elmasian, R., Galambos, R., Bernheim, A. (1980). Loudness enhancement and decrement in four paradigms. Journal of the Acoustical Society of America, 67, 601-607.

Greenberg, G. Z., \& LARKIN, W. D. (1968). Frequency-response characteristic of auditory observers detecting signals of a single frequency in noise: The probe-signal method. Joumal of the Acoustical Society of America, 44, 1513-1523.

HAFTER, E. R., \& SCHLAUCH, R. S. (1989). Factors in detection under uncertainty. Journal of the Acoustical Society of America, 86, S112.

Howard, J. H., Jr., O'Toole, A. J., Parasuraman, R., \& Bennett, K. B. (1984). Pattern-directed attention in uncertain-frequency detection. Perception \& Psychophysics, 35, 256-264.

LEEK, M. R. (1986). Directed attention in complex sound perception. In W. A. Yost \& C. S. Watson (Eds.), Auditory processing of complex sounds (pp. 278-288). Hillsdale, NJ: Erlbaum.

LEeK, M. R., \& Colleran, E. (1984). Individual differences in pattern discrimination by naive listeners. Journal of the Acoustical Society of America, 76, S14.

LEEK, M. R., WATSON, C. S. (1984). Learning to detect auditory pattern components. Journal of the Acoustical Society of America, 76, 1037-1044.

LEVITT, H. (1971). Transformed up-down methods in psychoacoustics. Journal of the Acoustical Society of America, 49, 467-477.

LUCE, R. D., \& GREEN, D. M. (1978). Two tests of a neural attention hypothesis for auditory psychophysics. Perception \& Psychophysics, 23, 363-371.

MCBRIDE, D. (1986). Rhythm and frequency factors in the perception of tone sequences. Unpublished master's thesis, Arizona State University.

MoOre, B. C. J., \& GlasberG, B. R. (1983). Suggested formulae for calculating auditory-filter bandwidths and excitation patterns. Journal of the Acoustical Society of America, 74, 750-753.

Patterson, R. D., \& MoORe, B. C. J. (1986). Auditory filters and excitation patterns as representations of frequency resolution. In B. C. J. Moore (Ed.), Frequency selectivity in hearing (pp. 123-177). New York: Academic Press.

Patterson, R. D., Nimmo-Smith, I., Weber, D. L., \& Milroy, R. (1982). The deterioration of hearing with age: Frequency selectivity, the critical ratio, the audiogram, and speech threshold. Journal of the Acoustical Society of America, 72, 1788-1803.

PolLACK, I. (1975). Auditory informational masking. Journal of the Acoustical Society of America, 57, 55.

Rabinowtz, W. M., Bilger, R. C., Trahiotis, C., \&uetzel, J. (1980). Two-tone masking in normal hearing listeners. Journal of the Acoustical Society of America, 68, 1096-1106.

SChARF, B. (1964). Partial masking. Acustica, 14, 16-23.

Scharf, B., Quigley, S., Aoki, C., Peachey, N., \& Reeves, A. (1987). Focused auditory attention and frequency selectivity. Perception \& Psychophysics, 42, 215-223.

SPIEGEL, M. F., \& Watson, C. S. (1981). Frequency discrimination with components of well-learned patterns. Journal of the Acoustical Society of America, 69, 223-230.

TAYLOR, M. M., \& Forbes, S. M. (1969). Monaural detection with contralateral cue (MDCC): I. Better than energy detector performance by human observers. Journal of the Acoustical Society of America, 46, 1519-1526.

Tougas, Y., \& Bregman, A. S. (1990). Auditory streaming and the continuity illusion. Perception \& Psychophysics, 47, 121-126.

VAN NOORDEN, L. P. A. S. (1975). Temporal coherence in the perception of tone sequences. Unpublished doctoral dissertation, Eindoven University of Technology, The Netherlands.

WatKINS, A. J., \& DYSON, M. C. (1985). On the perceptual organization of tone sequences and melodies. In P. Howell, I. Cross, \& R. West (Eds.), Musical structure and cognition (pp. 71-119). New York: Academic Press.

Watson, C. S. (1980). Time course of auditory perceptual learning. Annals of Otology, Rhinology \& Laryngology, 89(Suppl. 74), 96-102.

WATSON, C. S., FoYle, D. C. (1985). Central factors in the discrimination and identification of complex sounds. Journal of the Acoustical Society of America, 78, 375-380.

Watson, C. S., Kelly, W. J., Wroton, H. W. (1976). Factors in the discrimination of tonal patterns: II. Selective attention and learning under various levels of stimulus uncertainty. Journal of the Acoustical Society of America, 60, 1176-1185.

Watson, C. S., Wroton, H. W., Kelly, W. J., \& Benbasset, C. A. (1975). Factors in the discrimination of tonal patterns: I. Component frequency, temporal position, and silent intervals. Journal of the Acoustical Society of America, 57, 1175-1181.

Wier, C. C. , Jesteadt, W., Green, D. M. (1977). Frequency discrimination as a function of frequency and sensation level. Journal of the Acoustical Society of America, 61, 178-184.

(Manuscript received October 19, 1990; revision accepted for publication May 2, 1991.) 